\title{
Lawsone and Indigo Loaded Sepiolite Nanofibers for Hair Coloring with Sustained Release
}

Naureen Rahman ${ }^{1 \boxminus}$, Anik Karan*1冋, Yuri Lvov*1, Zhengzheng Liao ${ }^{2}$, Aakash Parekh², Ronak Rughani², Sivaramakrishnan Muthukrishnan*2

${ }^{1}$ Institute for Micromanufacturing, Louisiana Tech University, 505 Tech Dr., Ruston, LA 71272, USA

2 L'Oréal USA- Advanced Research, 133 Terminal Avenue, Clark, NJ 07066, USA

Correspondent authors: Y. Lvov, S. Muthukrishnan, and A. Karan, ylvov@latech.edu, siva.muthukrishnan@rd.loreal.com, anik1432@gmail.com
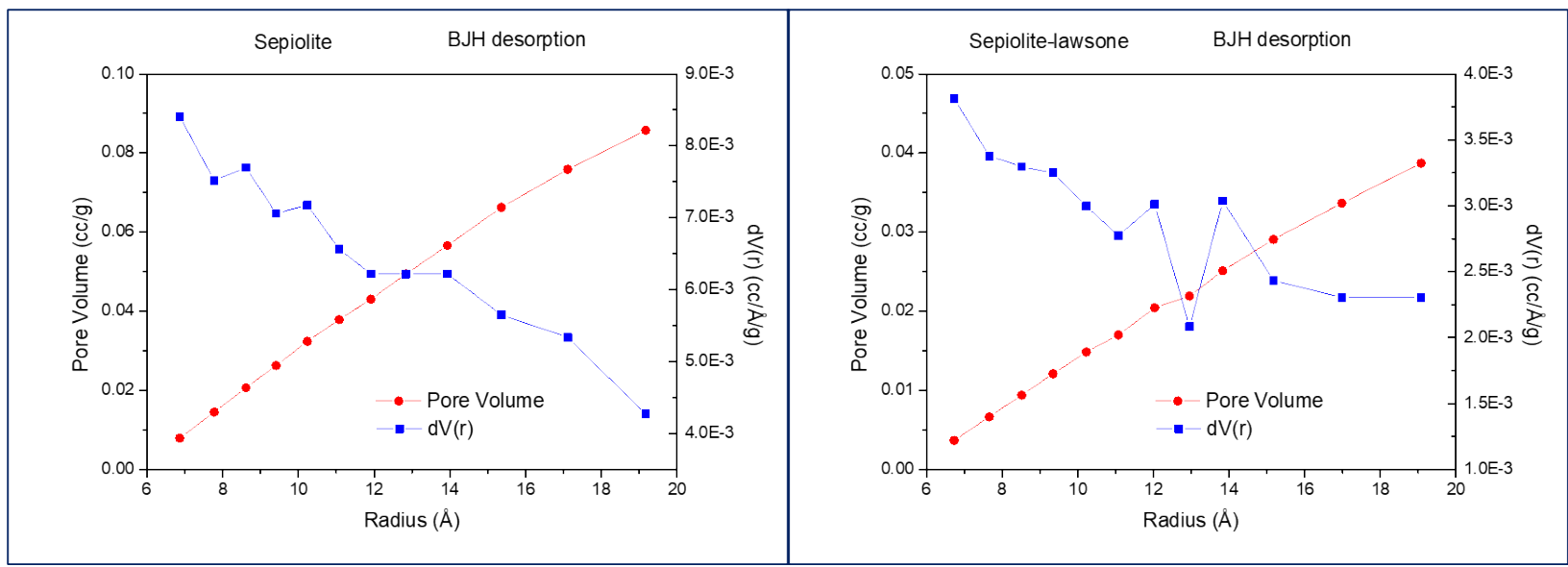

Figure S1: Desorption characteristics based on BET analysis of pristine sepiolite (left) and sepiolite-lawsone sample (right)

Table S2: Nanoclay pore size parameters based on BET analysis for indigo

\begin{tabular}{|l|c|c|}
\hline & Pristine sepiolite & Indigo-sepiolite \\
\hline Surface area, $\pm 1, \mathrm{~m}^{2} / \mathrm{g}$ & 151 & 75 \\
\hline Pore volume, $\pm 0.01, \mathrm{cc} / \mathrm{g}$ & 0.09 & 0.03 \\
\hline Pore radius $\mathrm{Dv}(\mathrm{r}), \pm 0.2, \mathrm{~nm}$ & 0.7 & 0.4 \\
\hline
\end{tabular}



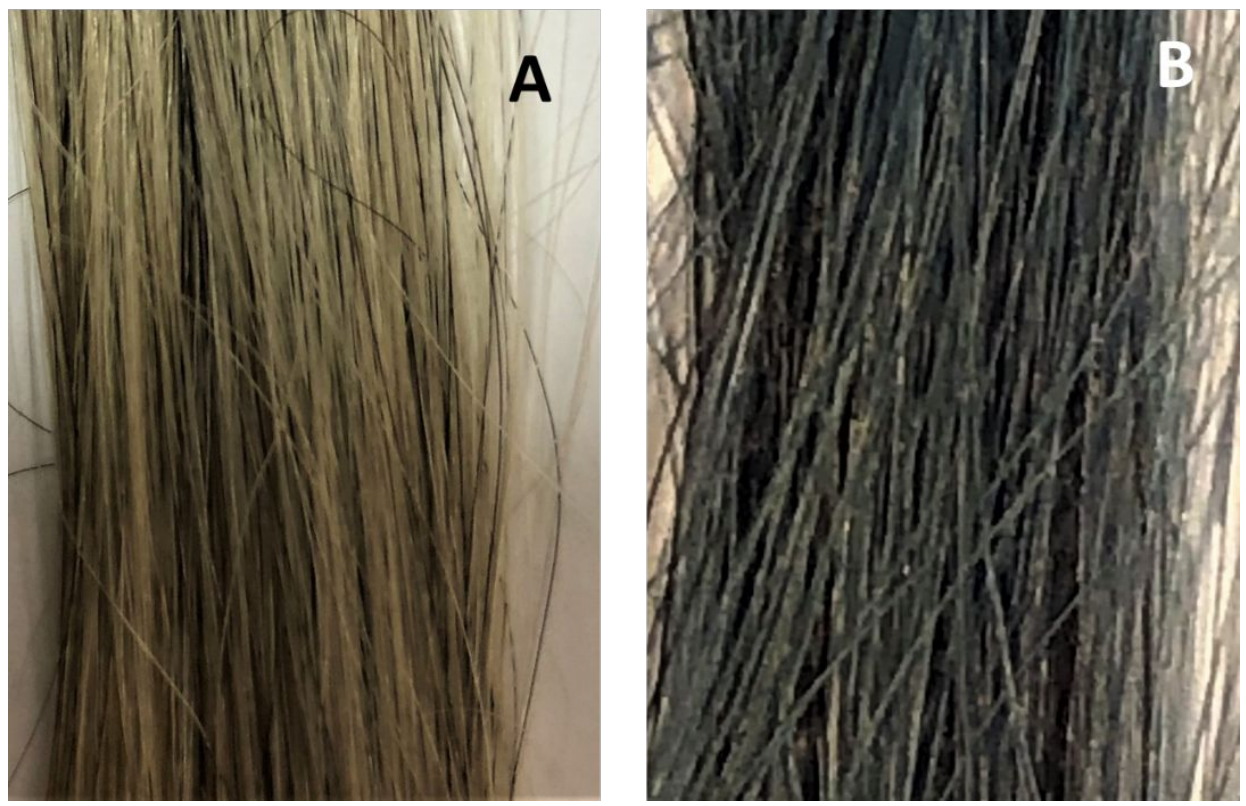

Figure S3: Image of virgin gray hair (A) and sepiolite-indigo coated hair (B)

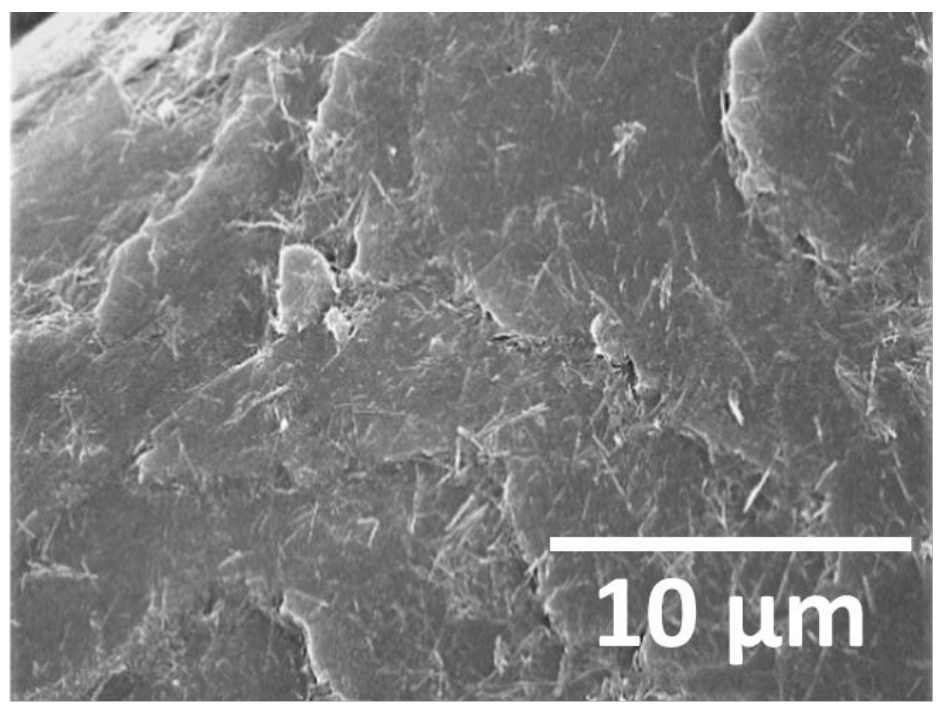

Figure S4: SEM image of sepiolite-indigo coated virgin hair 


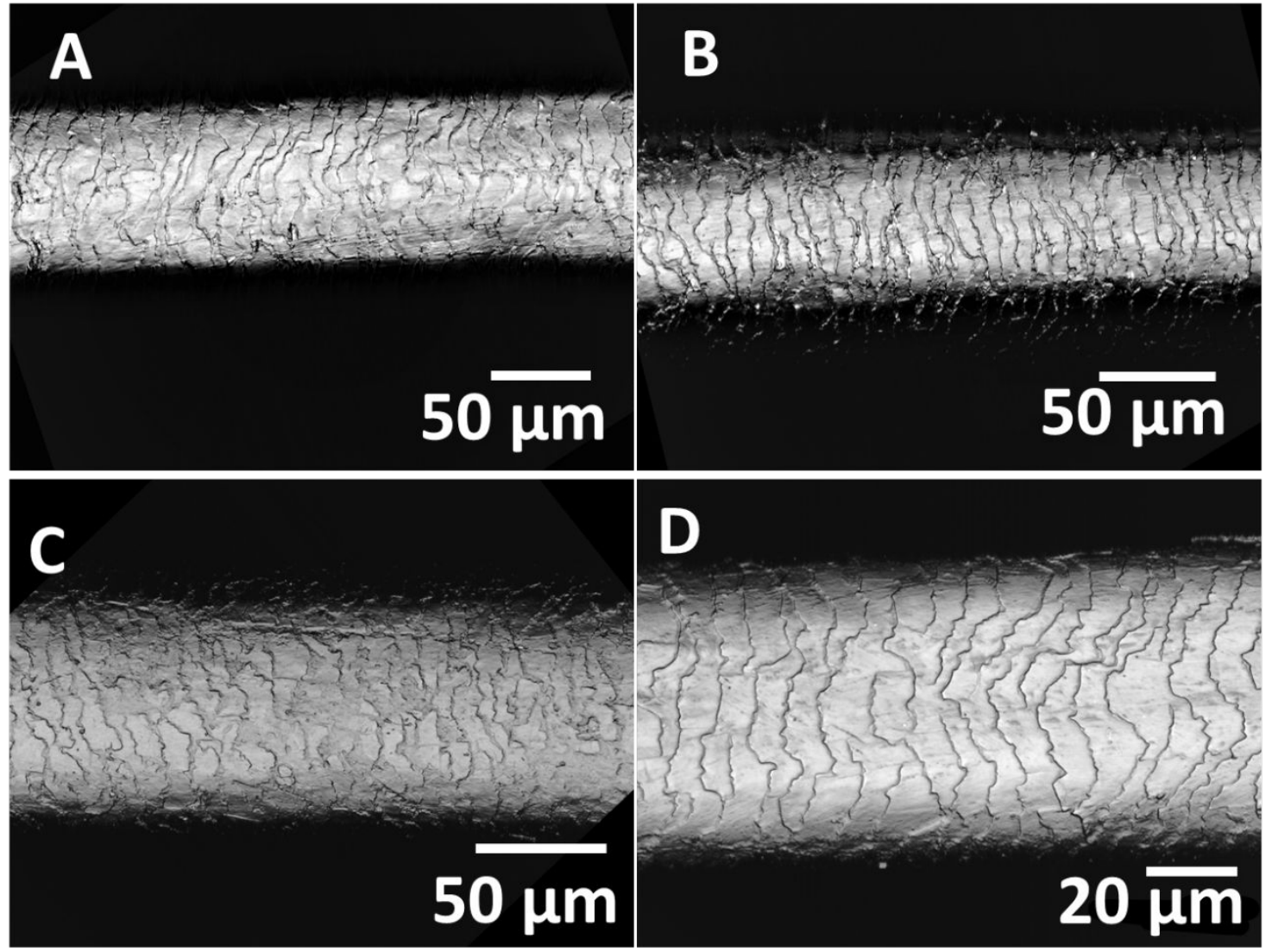

Figure S5: 3D measurement laser scanning microscopy of the virgin (a) and permed (b) untreated hair; virgin (c) and permed hair (d) treated by sepiolite-lawsone 


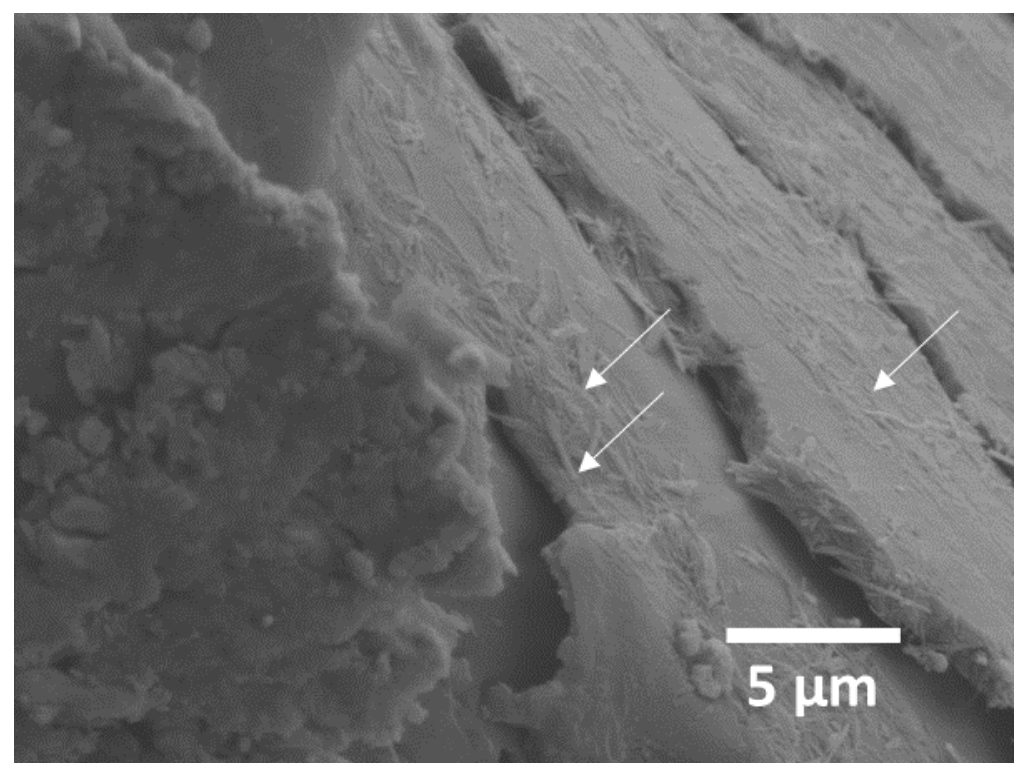

Figure S6: Cross-section SEM images of sepiolite-lawsone coated virgin hair, the arrow markes indicate the presence of sepiolite on the hair 